\title{
Pancreas Modeling from IVGTT Data Using A Deterministic Optimal Search Method
}

\author{
Dayu Lv \\ Department of Aerospace and Mechanical Engineering \\ University of Notre Dame \\ Notre Dame, IN 46556, U.S.A. \\ Email:dlv@nd.edu
}

\author{
Bill Goodwine \\ Department of Aerospace and Mechanical Engineering \\ University of Notre Dame \\ Notre Dame, IN 46556, U.S.A. \\ Email: jgoodwin@nd.edu
}

\begin{abstract}
Metabolism modeling has the potential to provide guidance on medicine manufacture and medical treatment in an efficient and economic way. A pancreas model developed from a mathematical description of the relevant physiology will demonstrate insulin dynamics and provide a platform for comparing metabolic abilities among different health conditions. As a part of whole body metabolism model, the pancreas model is composed of three compartments: insulin clearance $\left(I_{d}\right)$, generation $\left(I_{g}\right)$ and release into plasma $\left(I_{r}\right)$. Based on data from intravenous glucose tolerance tests (IVGTT) available in the literature, a deterministic optimal search method called DIRECT (DIviding RECTangles) was implemented to find the model parameters. Validation was performed on data sets that are diffrent from those used for the optimization.
\end{abstract}

Keywords-metabolism modeling;pancreas model;DIRECT;

\section{INTRODUCTION}

The health and quality of life problems associated with metabolic disorders such as diabetes and obesity are of increasing concern to society and hence an increasing focus area for researchers. According to the American Diabetes Association, the economic cost of diabetes in 2007 was approximately $\$ 174$ billion [1]. A mathematical model of the components of whole body metabolism would be of great benefit because it would enable simulations using computer models as a supplement to or replacement for traditional experimental methods. In human metabolic pathways, glucose and insulin are two of the primary substances maintaining and regulating daily energy needs. During the process of glucose uptake and transportation to peripheral locations for consumption or storage, insulin regulates its rates of delivery and related chemical reactions. As a whole, the network of glucose and insulin regulates an important part of the energy balance in the body. The parameters in mathematical models will reflect the complexity and characteristics of glucose and insulin kinetcis and difference in parameter ranges between normal and diabetic models may lead to important physiological insight.

Since the 1970s, Bergman and his coworkers developed a minimal model for the glucose and insulin metabolic system and proposed several parameters to represent glucose tolerance in humans, such as glucose effective rate, insulin sensitivity, etc. [2]-[7]. This model brought about the compartmental modeling concept with three variables: plasma glucose $(G)$, plasma insulin $(I)$ and remote insulin $(X)$. One of the drawbacks of the minimal model was that it described the metabolism empirically rather than mechanistically. Subsequently many analysis tools were developed based on the minimal model. For example, Vicini [8] utilized labeled glucose in the experiments. He expanded labeled glucose modeling into a two-compartment model and introduced three new metabolic indexes to represent the metabolic rates. Toffolo [9] improved upon this model afterward by assuming the insulin-independent glucose disposal as a fraction of steady-state glucose disposal. Monte Carlo and estimators were implemented in some analysis, [10], [11].

The minimal model was extended in various aspects. Jauslin [12] designed a model for oral glucose tolerance tests. Grodsky proposed a hypothesis of insulin heterogeneous storage with a bell shape threshold distribution of glucose [13], [14]. In other models, endogenous glucose release from liver and kindey was connected to the minimal model as additive compartments [15]-[18]. Heart compartment, compartment distribution volumes, $\beta$-cells weight, etc., were also incorporated in models as circulatory connections [19][23]. Among these models, most of them were constructed without validation.

In this paper we presented a pancreas model describing insulin dynamics based on its physiological characteristics. It reflected organs' ability to remove insulin in plasma, the glucose transportation ability via glucose transporters (GLUT2), the insulin productivity and membrane ion channels controllability of $\beta$-cells. This model was composed of three compartments: an exponential decay compartment $\left(I_{d}\right)$ representing the insulin clearance rate, a generation compartment $\left(I_{g}\right)$ representing insulin generation rate inside $\beta$-cells and a release compartment $\left(I_{r}\right)$ representing insulin releasing rate into plasma. Simulations were based on the data sets of IVGTT found in the literature. Ultimately this pancreas model will be incorporated into a whole body metabolism model in the future. Furthermore, the model parameters may imply different health statuses as a guide 
for medical analysis and medicine manufacture.

To find the parameters, we implemented a deterministic optimal search method, DIRECT, to obtain the minium of the object function [24], [25], which in the present case is a measure of the difference of the response of our model from a set of published experimental data. An advantage of this optimization method is that, while it is not guaranteed to find a global minimum, the search is global, and hence not restricted to finding only local minima. It defines a hypercube from the parameters and divides it into hyper-rectangles in every direction from the center. Then it calculates object functions at the centers of each hyper-rectangle, determines candidate hyper-rectangles called Potentially Optimal Rectangles (POR) through the algorithm of Gift Wrapping, and continues to divide these candidate PORs until the search criteria is achieved. The parallel computing environment is the module of MPICH-1.2.7. At the end, we validated the model from different IVGTT data sets.

\section{Mathematical Modeling}

\section{A. Model Establishment}

In this paper, the pancreas model describes insulin secretion and clearance rates, which are determined by glucose concentration, glucose transporters, $\beta$-cells' abilities of insulin generation and release. The three compartments: insulin generation $\left(I_{g}\right)$, insulin release $\left(I_{r}\right)$ and insuline clearance $\left(I_{d}\right)$ are illustrated in Figure 1.

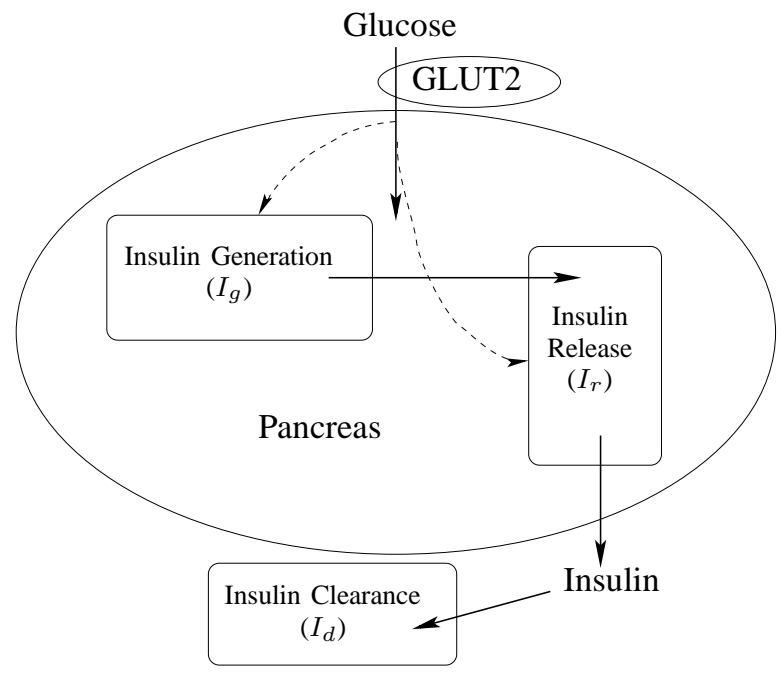

Figure 1. Proposed pancreas model.

Glucose enters $\beta$-cells faciliated by the transporters GLUT2, which has been described in the literature by Michaelis-Menten kinetics with $K_{m}=7-20 \mathrm{mM}$ [26]. The high $K_{m}$ reflects $\beta$-cells' sensitivity to different glucose concentrations. The rate of glucose entering $\beta$-cells ( $\mathrm{mmol} / \mathrm{min}$ ), is defined as

$$
\frac{d}{d t} G_{1}=V_{\max } \frac{[G]}{K_{1}+[G]},
$$

where $G_{1}$ (mmol) represents the amount of glucose entering $\beta$-cells; $V_{\max }\left(\mathrm{mmol} \cdot \mathrm{min}^{-1}\right)$ represents the maximum glucose removal rate; $[G](\mathrm{mM})$ represents the glucose concentration in plasma; and $K_{1}(\mathrm{mM})$ represents the MichaelisMenten constant. Since $V_{\max }$ can be combined with other parameters, as described subsequently, it is not included in the parameters space for the optimization.

After entering the membranes of the $\beta$-cells, glucose stimulates the synthesis of insulin which is then stored in vesicle granules. Proinsulin is the precursor of insulin synthesis. The ratio of proinsulin to insulin during a hyperglycemic clamp is decreasing and at the end acheives a constant level, illustrating the saturation of insulin generation under a specific glucose concentration [27]. A sigmoid relation between proinsulin synthesis and glucose concentration in rats was given in [28], [29]. So the insulin generation rate, $I_{g}\left(\mathrm{mU} \cdot \mathrm{min}^{-1}\right)$, is given by

$$
I_{g}=\frac{K_{2}}{1+e^{-K_{3} \times\left(G_{1}-K_{4}\right)}}+K_{5},
$$

where $G_{1}$ (mmol) represents the amount of glucose transported into $\beta$-cells; $K_{2}\left(\mathrm{mU} \cdot \mathrm{min}^{-1}\right)$ represents the difference between the maximal and basal generation rates; $K_{3}$ $\left(\mathrm{mmol}^{-1}\right)$ represents the glucose ability to stimulate insulin synthesis; $K_{4}(\mathrm{mmol})$ represents the glucose amount to stimulate the insulin generation rate increased to half of the maximal ability; and $K_{5}\left(\mathrm{mU} \cdot \mathrm{min}^{-1}\right)$ represents the basal insulin generation rate.

Glucose inside the $\beta$-cells is metabolized to generate energy, which may turn off the $\mathrm{K}^{+}$-ATP-dependent channels to produce membrane depolarization. This depolarization turns on calcium channels to stimulate insulin release into plasma [30], [31]. Insulin is released by exocytosis of the vesicle granules following a sigmoid function [32]. The insulin release rate, $I_{r}\left(\mathrm{mU} \cdot \mathrm{min}^{-1}\right)$, represents the dynamics of membrane ion channels and may be represented as follows

$$
I_{r}=\frac{K_{6}}{1+e^{-K_{7} \times\left(G_{1}-K_{8}\right)}}+K_{9}
$$

where $G_{1}(\mathrm{mmol})$ represents the amount of glucose transported into $\beta$-cells; $K_{6}\left(\mathrm{mU} \cdot \mathrm{min}^{-1}\right)$ represents the difference between the maximal and basal insulin release rates; $K_{7}\left(\mathrm{mmol}^{-1}\right)$ represents the ability of glucose to stimulate insulin release; $K_{8}(\mathrm{mmol})$ represents the glucose amount inside $\beta$-cells to trigger the insulin release rate increased to half of the maximal ability; and $K_{9}\left(\mathrm{mU} \cdot \mathrm{min}^{-1}\right)$ represents the basal insulin release rate.

The amount of insulin stored in $\beta$-cell granules, $I_{i}(\mathrm{mU})$, is defined in Table I where $I_{\text {iold }}(\mathrm{mU})$ represents current amount of insulin in granules; and $I_{\text {inew }}(\mathrm{mU})$ represents the amount of insulin in granules after a time interval $\Delta t$ (min). The reason for the definition is that, although many ion channels may open due to the high glucose concentration in plasma (which will carry more glucose into $\beta$-cells), there 
Table I

DEFINITION OF INSULIN AMOUNT IN GRANULES $\left(I_{i}\right)$ AND INSULIN RELEASE RATE $\left(I_{r}\right)$ FOR A TIME INTERVAL $\Delta t$.

\begin{tabular}{|l|l|}
\hline$I_{\text {iold }}>I_{r} \cdot \Delta t$ & $\begin{array}{l}I_{\text {inew }}=I_{\text {iold }}+\left(I_{g}-I_{r}\right) \cdot \Delta t \\
I_{r}=\text { Equation } 3\end{array}$ \\
\hline$I_{\text {iold }} \leqslant I_{r} \cdot \Delta t$ & $I_{\text {inew }}=I_{g} \cdot \Delta t$ \\
& $I_{r}=I_{\text {iold }} / \Delta t$ \\
\hline
\end{tabular}

may not be sufficient insulin in vesicle granules for release. Hence, the initial insulin storage amount, $K_{10}=I_{i 0}(\mathrm{mU})$, serves as another parameter in the model.

In the body, the pancreas releases insulin into plasma via the portal vein, $60 \%$ of which is immediately removed by the liver. After entering circulation, the kidney removes approximately $50 \%$ of peripheral insulin [31], [33]. Insulin is bound to receptors and most of it is degraded by the insulin-degrading enzymes in the intracellular space while the remaining insulin reenters plasma. The dynamics of insulin removal follow a Michaelis-Menten function [34], [35]. So the clearance rate $I_{d}\left(\mathrm{mU} \cdot \mathrm{l}^{-1} \cdot \mathrm{min}^{-1}\right)$ is

$$
I_{d}=-K_{11} \frac{I}{I+K_{12}},
$$

where $K_{11}\left(\mathrm{mU} \cdot 1^{-1} \cdot \mathrm{min}^{-1}\right)$ represents the maximal insulin removal rate; and $K_{12}\left(\mathrm{mU} \cdot \mathrm{l}^{-1}\right)$ represents the MichaelisMenten constant.

Finally the dynamic of insulin concentration is defined as:

$$
\frac{d}{d t} I=I_{d}+\frac{I_{r}}{V_{p}},
$$

where $V_{p}$ (1) represents the plasma volume. The volume of plamsa is determined from [36] assuming its value proportional to the subject's body weight. If a subject's weight was not provided, a standard of $70 \mathrm{~kg}$ was assumed.

\section{B. Optimal Search}

There are 12 parameters $\left(K_{i}, i=1, \cdots, 12\right)$ to be determined in the model. We adopted the experimental data from [3], [8], [17], [37]-[39] and divided them into two groups for optimization (4 data sets) and validation (3 data sets). In these experiments, IVGTTs were performed on normal subjects and the time span of each experiment was at least 180 minutes. The pooled samples of data sets in each group were implemented in simulations [40].

We used a deterministic optimal search method called DIRECT for optimization [24], [25]. One of its advantages is that it is a global search method in a multi-dimension parameters space with simple boundaries. Initially the parameters space is a unit hyper-cube with each side denoted as $\hat{K}_{i} \in[0,1]$, where $\hat{K}_{i}$ was a scale of the corresponding parameter $K_{i}=A_{i} \hat{K}_{i}$ with magnitude $A_{i}$. The coordinates of every point in this hyper-cube may be substituted into the pancreas model for simulations. To compare data from simulations and clinical experiments with a total sample size of $m$, we defined the objective function $f$ as following:

$$
f=\sqrt{\sum_{j=1}^{m}\left(\frac{x_{j}-x_{j 0}}{x_{j 0}}\right)^{2}}
$$

where $j \in[1, m]$ represents the sample size of the pooled samples data for optimization; and $x_{j}, x_{j 0}$ represent the corresponding data of simulation and pooled samples at the sample time point respectively. These data points $x_{j}, x_{j 0}$ represent plasma insulin levels at a given time and plasma glucose concentration.

\section{Results}

The simulation results of optimization and validation are demonstrated in Figure 2 with the determined parameters: $K_{1}=22.182 \mathrm{mM}, \quad K_{2}=44.460 \mathrm{mU} \cdot \mathrm{min}^{-1}$, $K_{3}=3150.211 \mathrm{mmol}^{-1}, K_{4}=3.074 \times 10^{-3} \mathrm{mmol}, K_{5}=44.155$ $\mathrm{mU} \cdot \mathrm{min}^{-1}, \quad K_{6}=180.262 \quad \mathrm{mU} \cdot \mathrm{min}^{-1}, \quad K_{7}=2026.597$ $\mathrm{mmol}^{-1}, K_{8}=2.945 \times 10^{-3} \mathrm{mmol}, K_{9}=9.892 \mathrm{mU} \cdot \mathrm{min}^{-1}$, $K_{10}=326.932 \mathrm{mU}, \quad K_{11}=88.897 \mathrm{mU} \cdot 1^{-1} \cdot \mathrm{min}^{-1}$, and $K_{12}=84.934 \mathrm{mU} \cdot \mathrm{l}^{-1}$. The validation proved that the pancreas model fitted the clinical data very well.

Furthermore, the simulation results of each data set (ID1, 2 , and 3 ) in the validation group are demonstrated respectively in Figure 3 showing that the pancreas model still can capture the dynamics of insulin response in individual expemeriments.

\section{CONCLUSION}

In this paper we presented a physiological pancreas model succesfully describing insulin dynamics in $\beta$-cells. Its parameters can be determined as a global optimum via DIRECT demonstrated in Figure 2 and 3. The optimization and validation results showed the agreement between the model and the clinical experiments. One of the model's advantages is its intrinsic ability of describing the pancreas physiological characteristics such as glucose transportation $\left(K_{1}\right)$, insulin generation $\left(K_{2}, K_{3}, K_{4}, K_{5}\right)$, release $\left(K_{6}, K_{7}, K_{8}, K_{9}\right)$, granules reservoir $\left(K_{10}\right)$ and removal $\left(K_{11}, K_{12}\right)$. The pancreas model not only can be applied to derive a set of parameters for a general normal subject as stated in this paper, but also it can record an individual's pancreas health dynamics. We can learn from the history of the parameters about how the characteristics of pancreas are changing which is benefit of long term metabolism analysis and provides guidance on medical research for finding pharmaceutic targets. Last but not least, this pancreas model can be connected as a component to a whole body metabolism model for extended research [41]. Since the insulin plays an important role in regulating energy in the whole body, its accurate representation will help understanding complex metabolic pathways. 

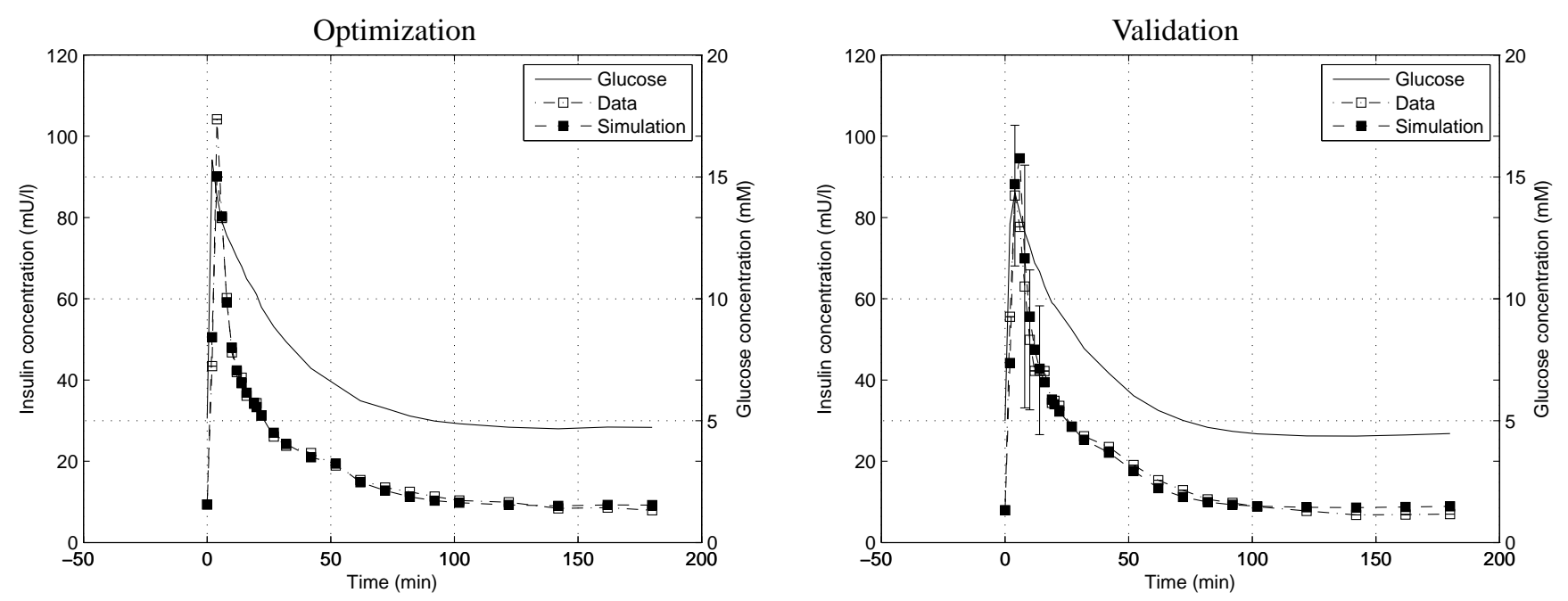

Figure 2. Simulation results of optimization and validation.

In the future, this pancreas model may be improved in serveral areas. First of all, insulin amount in pancreas granules varies for different individuals which might be obtained via non-invasive image techniques or determined by multiinjection of glucose or insulin. The different insulin release peaks reflects the insulin amount and generation ability. Secondly, the governing equation of insulin generation may be revised to a better function if more details obtained inside $\beta$-cells about this process. Thirdly as mentioned in some literatures [42]-[44], $\beta$-cells have a property of oscillation. We can extend our current model to a multi-scale problem to investigate the relation between smaller time scale oscillation and the insulin release in a larger time scale.

In summary, the model presented in this paper describes pancreas in a physiological way. The model parameters were acheived via a deterministic global optimal search. Simulation results agreed with experimental data. This model might be used for describing health situation for a general group of subjects or for an individual which is beneficial for medical research. Also it can be expanded for further physiological modeling.

\section{ACKNOWLEDGMENT}

Support from the Notre Dame Faculty Research Program is gratefully acknowledged.

\section{REFERENCES}

[1] A. D. Associationn, "http://www.diabetes.org/diabetesstatistics.jsp."

[2] R. Bergman et al., "Quantitative estimation of insulin sensitivity," Am. J. of Physiology, vol. 236, pp. E667-677, 1979.
[3] - "Physiologic evaluation of factors controlling glucose tolerance in man," J. Clin. Invest., vol. 68, pp. 1456-1467, 1981.

[4] R. Bergman, "Toward physiological understanding of glucose tolerance: Minimal-model approach," Diabetes, vol. 38, no. $12,1989$.

[5] C. Cobelli and G. Toffolo, "A model of glucose kinetics and their control by insulin, compartmental and noncompartmental approaches," Mathematical Biosciences, vol. 72, pp. $291-$ 315,1984

[6] G. Pacini and R. Bergman, "MINMOD: A computer program to calculate insulin sensitivity and pancreatic responsibility from the frequently sampled intravenous glucose tolerance test," Computer Methods and Programs in Biomedicine, vol. 23, pp. 113-122, 1986.

[7] G. Toffolo et al., "Quantitative estimation of beta cell sensitivity to glucose in the intact organism: A minimal model of insulin kinetics in the dog," Diabetes, vol. 29, pp. 979-990, 1980.

[8] P. Vicini et al., "The hot IVGTT two-compartment minimal model: Indexes of glucose effectiveness and insulin sensitivity," Am. J. Physiol., vol. 273, pp. 1024-1032, 1997.

[9] G. Toffolo and C. Cobelli, "The hot IVGTT two-compartment minimal model: an improved version," Am. J. Physiol. Endocrinol. Metab., vol. 284, pp. E317-E321, 2003.

[10] T. Lotz et al., "Monte Carlo analysis of a new model-based method for insulin sensitivity testing," Computer Methods and Programs in Biomedicine, vol. 89, pp. 215-225, 2008.

[11] C. Neatpisarnvanit and J. Boston, "Estimation of plasma insuliin from plasma glucose," IEEE Trans. on Biomed. Engr., vol. 49, no. 11, pp. 1253-1259, 2002. 

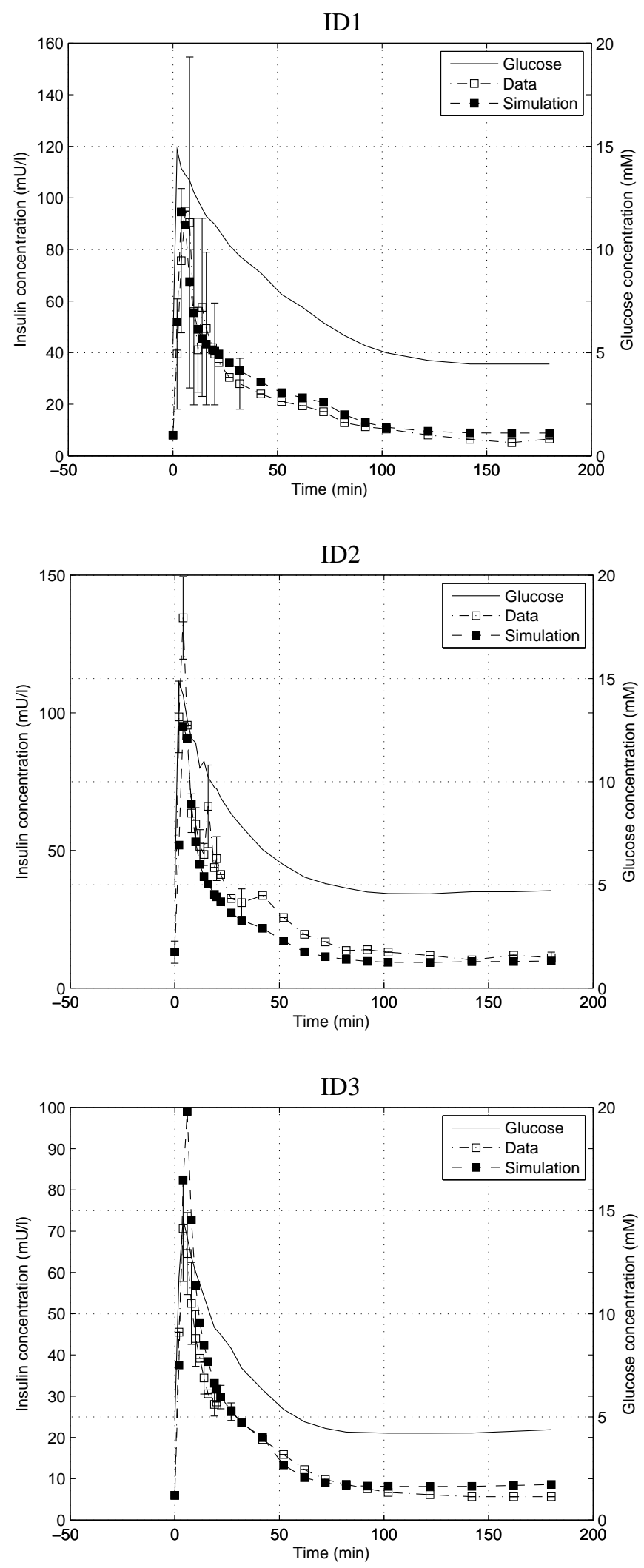

Figure 3. Simulation results of three data sets in the group of validation respectively.
[12] P. Jauslin et al., "An integrated glucose-insulin model to describe oral glucose tolerance test data in type 2 diabeteics," J. Clin. Pharmacol, vol. 47, pp. 1244-1255, 2007.

[13] G. Grodsky, "A threshold distribution hypothesis for packet storage of insulin and its mathematical modeling," J. Clin. Invest., vol. 51, pp. 2047-2059, 1972.

[14] R. Overgaard and O. Arino, "Mathematical beta cell model for insulin secretion following IVGTT and OGTT," Annals of Biomedical Engineering, vol. 34, no. 8, pp. 1343-1354, 2006.

[15] A. Caumo and C. Cobelli, "Hepatic glucose production during the labeled IVGTT: Estimation by deconvolution with a new minimal model," Am. J. Physiol., vol. 264, pp. E829-E841, 1993.

[16] P. Vicini et al., "Glucose effectiveness and insulin sensitivity from the minimal models: Consequences of undermodeling assessed by monte carlo simulation," IEEE Trans. on Biomed. Engr., vol. 46, no. 2, pp. 130-137, 1999.

[17] R. Hovorka et al., "Partitioning glucose distribution/transport, disposal, and endogenous production during IVGTT," Am. J. Physiol. Endocrinol. Metab., vol. 282, pp. E992-1007, 2002.

[18] K. Krudys et al., "Integrated model of hepatic and peripheral glucose regulation for estimation of endogenous glucose production during the hot IVGTT," Am. J. Physiol. Endocrinol. Metab., vol. 288, pp. E1038-E1046, 2005.

[19] A. Mari, "Assessment of insulin sensitivity and secretion with the labelled intravenous glucose tolerance test: improved modelling analysis," Diabetologia, vol. 41, pp. 1029-1039, 1998.

[20] B. Topp et al., "A model of $\beta$-cell mass, insulin, and glucose kinetics: Pathways to diabetes," J. Theor. Biol., vol. 206, pp. 605-619, 2000.

[21] T. Lotz et al., "Transient and steady-state euglycemic clamp validation of a model for glycemic control and insulin sensitivity testing," Diabetes Technology and Therapeutics, vol. 8, no. 3, pp. 338-346, 2006.

[22] H. Silber et al., "An integrated model for glucose and insulin regulation in healthy volunteers and type 2 diabetic patients following intravenous glucose provocations," J. Clin. Pharmacol, vol. 47, pp. 1159-1171, 2007.

[23] M. Koschorreck and E. Gilles, "Mathematical modeling and analysis of insulin clearance in vivo," BMC Systems Biology, vol. 2, p. 43, 2008.

[24] D. Jones et al., "Lipschitzian optimization without the lipschitz constant," J. of Optimization Theory and Application, vol. 79, no. 1, pp. 157-181, 1993.

[25] J. He et al., "Dynamic data structures for a direct search algorithm," Computational Optimization and Application, vol. 23, pp. 5-25, 2002.

[26] K. Frayn, Metabolic Regulation: A Human Perspective, 2nd ed. Oxford: Blackwell Science Ltd, 2003. 
[27] R. Weiss et al., " $\beta$-cell function across the spectrum of glucose tolerance in obese youth," Diabetes, vol. 54, pp. 1735-1743, 2005.

[28] S. Ashcroft et al., "The effect of sugars on (pro)insulin biosynthesis," Biochem J., vol. 174, pp. 517-526, 1978.

[29] F. Schuit et al., "Glucose stimulates proinsulin biosynthesis by a dose-dependent recruitment of pancreatic beta cells," Proc. Natl. Acad. Sci. USA, vol. 85, pp. 3865-3869, 1988.

[30] C. Cobelli et al., "Assessment of $\beta$-cell function in humans, simultaneously with insulin sensitivity and hepatic extraction, from intravenous and oral glucose tests," Am. J. Physiol. Endocrinol. Metab., vol. 293, pp. E1-15, 2007.

[31] G. Wilcox, "Insulin and insulin resistance," Clin. Biochem., vol. 26, pp. 19-39, 2005.

[32] D. Harrison et al., "Properties of isolated human islets of langerhans: insulin secretion, glucose oxidation and protein phosphorylation," Diabetologia, vol. 28, pp. 99-103, 1985.

[33] W. Duckworth et al., "Insulin degradation: Progress and potential," Endocrine Reviews, vol. 19, no. 5, pp. 608-624, 1998.

[34] J. Chase et al., "Adaptive bolus-based targeted glucose regulation of hyperglycaemia in critical care," Medical Engineering \& Physics, vol. 27, pp. 1-11, 2005.

[35] B. Thorsteinsson, "Kinetic models for insulin disappearance from plasma in man," Danish Medical Bulletin, vol. 37, no. 2, 1990.
[36] D. Levitt, "The pharmacokinetics of the interstitial space in humans," BMC Clinical Pharmacology, vol. 3, p. 3, 2003.

[37] J. Henriksen et al., "Increased glucose effectiveness in normoglycemic but insulin-resistant relatives of patients with noninsulin-dependent diabetes mellitus: a novel compensatory mechanism,” J. Clin. Invest., vol. 94, pp. 1196-1204, 1994.

[38] A. Avogaro et al., "Stable-label intravenous glucose tolerance test minimal model," Diabetes, vol. 38, pp. 1048-1055, 1989.

[39] G. Pacini et al., "Insulin ssensitivity and glucose effectiveness: minimal model analysis of regular and insulin-modified FSIGT," Am. J. Physiol., vol. 274, pp. E592-E599, 1998.

[40] P. Dunn, Measurement and data analysis for engineering and science. Boston: McGraw-Hill Higher Education, 2005.

[41] D. Lv and B. Goodwine, "A new metabolism model for human skeletal muscle," in BIODEVICES (2), 2008, pp. 238243.

[42] T. Chay and J. Keizer, "Minimal model for membrane oscillations in the pancreatic $\beta$-cell," Biophys. J., vol. 42, pp. 181-190, 1983.

[43] J. Sturis et al., "Computer model for mechanisms underlying ultradian oscillations of insulin and glucose," Am. J. Physiol., vol. 260, pp. E801-E809, 1991.

[44] I. Tolić et al., "Modeling the insulin-glucose feedback system: the significance of pulsatile insulin secretion," J. Theor. Biol. vol. 207, pp. 361-375, 2000. 